\title{
T cell receptor (TCR)-transgenic CD8 lymphocytes rendered insensitive to transforming growth factor beta (TGF $\beta$ ) signaling mediate superior tumor regression in an animal model of adoptive cell therapy
}

\author{
Jon G Quatromoni ${ }^{1}$, Yue Wang ${ }^{1}$, Dan D Vo ${ }^{1}$, Lilah F Morris ${ }^{1}$, Ali R Jazirehi ${ }^{1}$, William McBride ${ }^{2}$, Talal Chatila ${ }^{3}$, \\ Richard C Koya ${ }^{1}$ and James S Economou ${ }^{1,4,5,6^{*}}$
}

\begin{abstract}
Tumor antigen-reactive T cells must enter into an immunosuppressive tumor microenvironment, continue to produce cytokine and deliver apoptotic death signals to affect tumor regression. Many tumors produce transforming growth factor beta (TGF $\beta$ ), which inhibits T cell activation, proliferation and cytotoxicity. In a murine model of adoptive cell therapy, we demonstrate that transgenic Pmel-1 CD8 T cells, rendered insensitive to TGF $\beta$ by transduction with a TGF $\beta$ dominant negative receptor II (DN), were more effective in mediating regression of established B16 melanoma. Smaller numbers of DN Pmel-1 T cells effectively mediated tumor regression and retained the ability to produce interferon- $\gamma$ in the tumor microenvironment. These results support efforts to incorporate this DN receptor in clinical trials of adoptive cell therapy for cancer.
\end{abstract}

\section{Background}

Metastatic melanoma continues to be a therapeutic challenge. Adoptive cell therapy (ACT) using tumor-infiltrating lymphocytes (TILs) or T cell receptor-engineered lymphocytes has produced increased response rates, many clinically dramatic, but most are partial and patients generally relapse within a short time frame [1-3]. Factors contributing to these partial responses include down-regulation of $\mathrm{MHC}$ and antigen presentation by tumor cells, their resistance to $\mathrm{T}$ cell delivered death signals, and tumor production of immunosuppressive factors such as vascular endothelial growth factor (VEGF), Indoleaminepyrrole 2,3-dioxygenase (IDO), IL10 and transforming growth factor-beta (TGF $\beta)[4,5]$.

\footnotetext{
* Correspondence: jeconomou@mednet.ucla.edu

'Departments of Surgery, University of California, Los Angeles, CA 90095, USA

${ }^{4}$ Molecular and Medical Pharmacology, University of California, Los Angeles, CA 90095, USA

Full list of author information is available at the end of the article
}

Many reports have shown that melanoma and other cancers produce TGF $\beta$ which in turn promotes tumor invasion, metastasis and creates an immunosuppressive microenvironment that inhibits immune effector function [6,7]. TGF $\beta$ inhibits $\mathrm{T}$ cell activation, proliferation, cytotoxicity and promotes $\mathrm{T}$ regulatory cell inhibitory functions [8-11].

Different strategies to circumvent the inhibitory effects of TGF $\beta$ have been employed with varying success. The use of a dominant-negative TGF $\beta$ receptor II $(\mathrm{DN})$ as a decoy receptor to render $\mathrm{T}$ cells insensitive to TGF $\beta$ signaling allows them to retain proliferative and cytotoxic functions in the presence of exogenous TGF $\beta$ and to acquire resistance to inhibition by $\mathrm{T}$ regulatory cells (Treg) [12-15]. Transgenic mice with TGF $\beta$ insensitive $\mathrm{T}$ cells are resistant to lymphoma and melanoma tumor challenge [16].

In this study, we used an animal model of ACT in which antigen-reactive $T$ cells were rendered insensitive to TGF $\beta$ through transduction with a DN TGF $\beta$ RII transgene. These TGF $\beta$-insensitive, antigen-specific $T$ 
cells mount a more effective anti-tumor response towards B16 melanoma, most likely due to the ability of these effector cells to remain active in the tumor microenvironment.

\section{Materials and methods \\ Mice and cell lines}

C57BL/6 (The Jackson Laboratory) and Pmel-1 (kind gift from Dr. Nicholas Restifo, Surgery Branch, National Cancer Institute) [17] mice were bred and kept under defined-flora pathogen-free conditions at the American Association for Laboratory Animal Care-approved Animal Facility of the Division of Experimental Radiation Oncology, University of California, Los Angeles. The B16 murine melanoma cell line (American Type Culture Collection) was maintained in DMEM (Mediatech) with $10 \%$ FCS (Omega Scientific) and 1\% (v/v) penicillin, streptomycin, and amphotericin (Omega Scientific).

\section{Western blot analysis for p-SMAD2 expression}

Pmel-1 T cells were transduced with DN TGF $\beta$ RII (DN) retrovirus and sorted by FACS to ensure $>98 \%$ TGF $\beta$ RII expression. Untransduced and DN-transduced pmel-1 cells were incubated in $10 \mathrm{ng} / \mathrm{ml}$ TGF $\beta$ for $0,0.5,1,6$, $24 \mathrm{~h}$ prior to protein extraction. Cells were lysed at $4^{\circ} \mathrm{C}$ in radioimmuno-precipitation assay (RIPA) buffer [50 mM Tris-HCl (pH 7.4), 1\% NP-40, 0.25\% sodium deoxycholate, $150 \mathrm{mM} \mathrm{NaCl}$ ] supplemented with one tablet of protease inhibitor cocktail (Complete Mini; Roche) and phosphatase inhibitors (Santa Cruz Biotechnology, Santa Cruz, CA). A detergent-compatible protein assay kit (Bio-Rad, Hercules, CA) was used to determine protein concentration. An aliquot of total protein lysate was diluted in an equal volume of 2X SDS sample buffer, boiled for $5 \mathrm{~min}$, and cell lysates were electrophoresed on $10 \%$ SDS-PAGE gels. Western blot was carried out with anti-phospho-Smad 3 antibody (Cell Signaling Technology, Danvers, MA) at 1:500 overnight at $4 \mathrm{C}$, anti-total-Smad 3 antibody (Cell Signaling) at 1:1000 overnight at $4 \mathrm{C}$, and anti- $\beta$-Actin-HRP antibody (Sigma, St. Louis, MO) at 1:20,000 for $2 \mathrm{~h}$ at room temperature. Secondary anti-rabbit-HRP (Santa Cruz) was used at 1:10,000 for $30 \mathrm{~min}$.

\section{Retrovirus production and transduction of pmel-1 splenocytes}

The vector containing the dominant negative TGF $\beta$ receptor II, which encodes a truncated receptor lacking the entire kinase domain and most of juxta-membrane region was constructed by $\mathrm{PCR}$ amplification, using the TGF $\beta$ receptor II cDNA as template [18]. Primers were designed to include a stop codon after nucleotide 597, which corresponds to the 10th cytoplasmic codon region, flanked by Sal I and Bam HI sites. Amplified fragment was then inserted into the retroviral vector MSCV. DN TGF $\beta$ RII retrovirus was produced as previously described [19]. T cells were transduced 48 hours following polyclonal activation with anti-CD3 and antiCD28 (BD Bioscience) coated plates. $2.5 \times 10^{6}$ cells per well were transduced in $3 \mathrm{ml}$ total volume of virus and culture media in retronectin coated 24-well plates. Cells were spinnoculated twice by centrifugation at $1000 \times g$ for $2 \mathrm{hr}$ at $32^{\circ} \mathrm{C}$.

\section{Bone marrow-derived dendritic cells}

The generation of dendritic cells from murine bone marrow in cultures of granulocyte-macrophage colonystimulating factor (GM-CSF) and interleukin (IL)-4 was described previously [20]. Day 8 dendritic cell cultures were pulsed for $2 \mathrm{~h}$ with $1 \mu \mathrm{g} / \mathrm{ml}$ gp $100_{25-33}$ peptide for the Pmel-1 adoptive transfer model. Between $5 \times 10^{5}$ and $1 \times 10^{6}$ cells per mouse were injected s.c. in the right flank.

\section{Pmel-1 adoptive transfer therapy in vivo model}

B16 tumors were implanted s.c. as described previously [20]. When tumors reached 5 to $8 \mathrm{~mm}$ in diameter, mice received a myeloablating regimen of 900 cGy total body irradiation. The following day, Pmel-1 splenocytes were adoptively transferred into 8 experimental mice per group via a lateral tail vein. Subsequently, gp $100_{25-33}$ peptide-pulsed dendritic cells were given s.c. on the day of adoptive transfer and 1 week later, in both cases, followed by 3 days of daily i.p. administration of 50,000 IU IL-2. For co-adoptive transfer model, mock and DNtransduced Pmel-1 were added and mixed at 1:1 ratio prior to adoptive transfer of $10^{6}$ activated Pmel-1 followed by two rounds of IL-2 administration.

\section{Flow cytometry analysis}

Splenocytes and tumor-infiltrating lymphocytes, obtained from enzymatically digested B16 tumors harvested from mice as described previously [21], were stained with antibodies to $\mathrm{CD} 8 \alpha^{\mathrm{FITC}}$, TGF $\beta$ RII ${ }^{\mathrm{PE}}$, Thy $1.1^{\text {PerCP }}$, and $\mathrm{CD} 3^{\mathrm{APC}-\mathrm{Cy} 7}$ (BD Bioscience), and analyzed with a FACSCalibur machine using FCS Express software (DeNovo Software). Cells were initially gated on live cells area by FSC $x$ SSC analysis, then gated the CD3 positive/CD8 positive/Thy1.1 positive (staining for pmel-1 T cells), followed by TGF $\beta$ RII levels analysis. Intracellular IFN- $\gamma$ staining was done as described previously [22]. Briefly, 1 million cells were stimulated with $1 \mu \mathrm{M}$ specific peptide $\left(\operatorname{gp} 100_{(25-33)}\right)$ or non-relevant peptide Ovalbumin, plus brefeldin A (BD Pharmingen) and $50 \mathrm{U} / \mathrm{ml} \mathrm{IL-2,} \mathrm{for} \mathrm{six} \mathrm{hours} \mathrm{at} 37^{\circ} \mathrm{C}$ in $5 \% \mathrm{CO} 2$. Cells were then washed with staining buffer (PBS with $3 \%$ FBS and $0.09 \%$ sodium azide), pre-treated with antiFcR Ab for $10 \mathrm{~min}$, and then stained with anti-CD4, 
anti-CD8, and anti-Thy1.1 (BD Pharmingen) on ice for $30 \mathrm{~min}$. Cells were then permeabilized and fixed with Cytofix/CytoPerm (BD Pharmingen), then stained for intracellular IFN- $\gamma$ with anti-IFN- $\gamma$ or a isotype control mAb.

\section{Results}

\section{Pmel-1 CD8 T cells can be transduced to high efficiency} with a DN TGF $\beta$ retrovirus

The retroviral vector encoding the DN TGF $\beta$ RII (DN), in which the intracellular signaling sequence was deleted, is depicted in Figure 1A. Activated Pmel-1 splenocytes can be transduced to high efficiency (70-90\%) with this vector. Shown in Figure 1B are DN-transduced and mock-transduced Pmel-1 splenocytes stained with an antibody for the human (not murine) TGF $\beta$ RII receptor. The right hand panel (DN Pmel-1) shows the levels of enrichment of human DN receptor transgene after transduction. This DN TGF $\beta$ receptor has been shown in previous studies to inhibit TGF $\beta$ signaling. Pmel-1 T cells, transduced with the DN receptor, did not phosphorylate SMAD3 after incubation with exogenous TGF $\beta 1$ (Figure 1C). The proliferation of mocktransduced, but not DN-transduced, Pmel-1 cells was inhibited after exposure to TGF $\beta 1$ (Figure 1D). These results confirm that this $\mathrm{DN}$ receptor inhibits the antiproliferative effects of TGF $\beta$ (12).

\section{DN TGF $\beta$-transduced pmel-1 more effectively mediate B16 tumor regression}

Pmel-1 CD8 splenocytes express a transgenic TCR that recognizes gp $100_{25-33}$ in the context of $\mathrm{H}-2 \mathrm{D}^{\mathrm{b}}$; adoptive transfer of activated Pmel-1 can mediate partial or complete regression of established B16 melanoma in various animal tumor models [23,24]. The B16 melanoma used in these experiments produced about $1 \mu \mathrm{g}$ TGF $\beta / 10^{6}$ tumor cells $/ 24 \mathrm{hr}$. C57BL/ 6 mice bearing small-established B16 tumors $\left(\sim 64 \mathrm{~mm}^{3}\right)$ experienced significant delay in tumor outgrowth upon adoptive transfer of activated Pmel-1 T cells (Figure 2). A myeloablative conditioning regimen of 900 cGy whole body irradiation (accompanied by bone marrow rescue) creates space in the secondary lymphoid organs enabling better repopulation by administered $\mathrm{T}$ cells. Administration of IL-2 and/or gp $100_{25-33}$ peptide pulsed dendritic cell vaccine $\left(\mathrm{gp}_{100_{25-33}} / \mathrm{DC}\right)$ supports the expansion and antitumor activity of administered Pmel-1 T cells. Figure 2 depicts a direct comparison between DN-transduced (80\% transduction efficiency) and mock transduced Pmel-1; $10^{5}$ DN Pmel-1 produced greater tumor suppression than $10^{6}$ mock Pmel-1. These animals were supported by IL-2 administration alone.

\section{DN TGF $\beta$ effector T cell function with IL-2 and/or gp $100_{25-33} / \mathrm{DC}$ support}

A comparable set of experiments is shown in Figure 3 in which DN Pmel-1 or mock Pmel-1 were administered to B16 tumor-bearing mice that then received IL-2, gp100 $25-33$ /DC, both or neither supporting intervention. In Figure 3A, Pmel-1 were administered to conditioned mice with established B16 tumors; the DN group delayed tumor outgrowth to day 60 with neither IL-2 nor DC vaccine. The administration of IL-2 (3B), gp100 $25-33 / \mathrm{DC}$ (3 C) or IL-2/DC (3D) clearly enhances the antitumor activity of both mock and DN Pmel-1. DN Pmel-1 cells have superior anti-tumor biology in vivo compared to mock transduced Pmel in all groups except those animals receiving IL-2/DC in which tumor suppression for all Pmel treated mice extends to day 60 . These data are represented in Kaplan-Meier plots in Figure $3 \mathrm{E}$ where a clear pattern emerges. Control mice all die by day 24 . Only $11 \%$ of mock-transduced Pmel treated mice survived to day 120 whereas $53 \%$ of DNtransduced Pmel mice were still alive.

\section{Tumor infiltrating DN TGF $\beta$ pmel-1 T cells remain activated in the tumor microenvironment}

DN and mock-transduced Pmel-1 were co-adoptively transferred to B16 tumor-bearing and non tumorbearing mice; we wanted to compare their relative expansion, tumor infiltration, and activation in vivo. Tumor infiltrating and spleen repopulating Pmel-1 were retrieved at various intervals (days 14, 21, 28 post ACT) and evaluated for relative number and percent of cells producing IFN- $\gamma$ (by intracellular cytokine staining). There was not a reproducible preferential expansion or infiltration of either Pmel-1 population in the tumor or spleen, based on total cells counts analyzed from the harvested tissues (Additional file 1: Figure S1). However, in replicate experiments, a higher percentage of DN Pmel-1 retrieved from B16 tumors stained for IFN- $\gamma$. As shown in Figure $4 \mathrm{~B}$, the differences were very significant at all time points for tumor infiltrating Pmel-1, with DN population having a several fold higher percentage of IFN- $\gamma$ production. Spleen repopulation with the DN Pmel-1/mock Pmel-1 co-adoptive transfer also demonstrated higher IFN- $\gamma$ production among DN Pmel-1 on days 14 and 21 but not 28 , in both naïve and B16 tumor-bearing mice, and to a comparable degree. Analysis of spleen repopulation suggests that DN Pmel-1, in general, can maintain a higher level of Th1 cytokine production upon adoptive transfer into conditioned hosts. A remarkable comparison is between spleen and tumorinfiltrating Pmel-1, and the profound suppression of cytokine production in the latter population. These findings suggest that DN Pmel are functionally more active within the tumor microenvironment. 


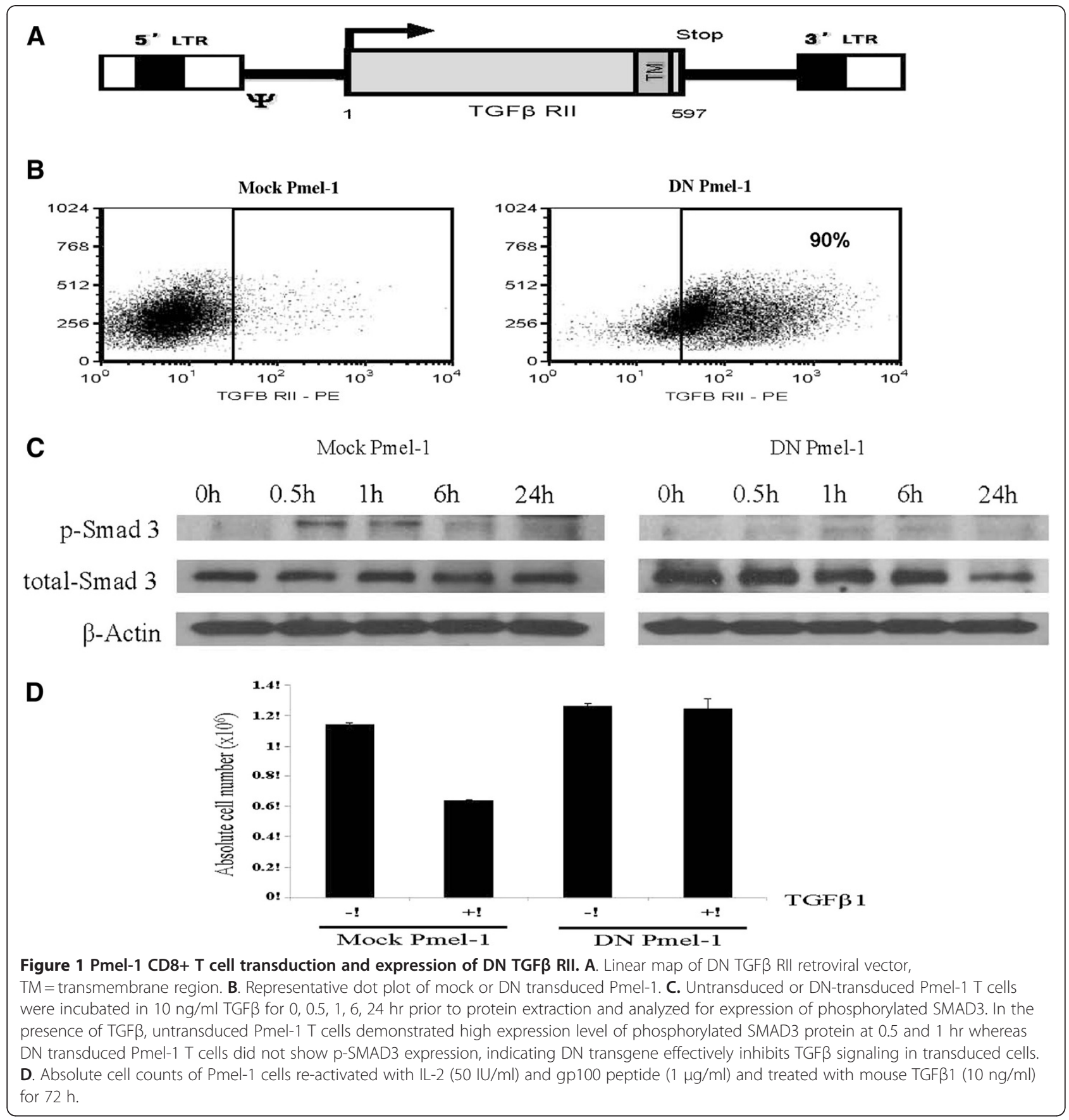

\section{Discussion}

We have shown that TCR-transgenic $\mathrm{T}$ cells transduced with a TGF $\beta$ dominant-negative receptor have superior anti-tumor activity against B16 melanoma in a model of adoptive cell therapy. This work extends the findings of several other groups using an identical DN receptor in a variety of models of auto-immunity and tumor immunity. The novelty of this confirmatory report is the use of TCR-transgenic $\mathrm{T}$ cells that recognize a self tumor antigen in a commonly used preclinical animal model. DN TGF $\beta$-transduced Pmel1 , on a cell for cell basis, were more than ten-fold more potent in mediating tumor regression of established tumors. We observed complete and durable regression in some mice receiving DN Pmel-1 with neither IL-2 nor DC vaccine support. Pmel-1 CD8 T cells retrieved from B16 tumor microenvironment are immunosuppressed; fewer than $10 \%$ express IFN- $\gamma$ by intracellular cytokine staining. In contrast, $25-35 \%$ of tumor-infiltrating DN Pmel-1 continued to produce IFN- $\gamma$, 


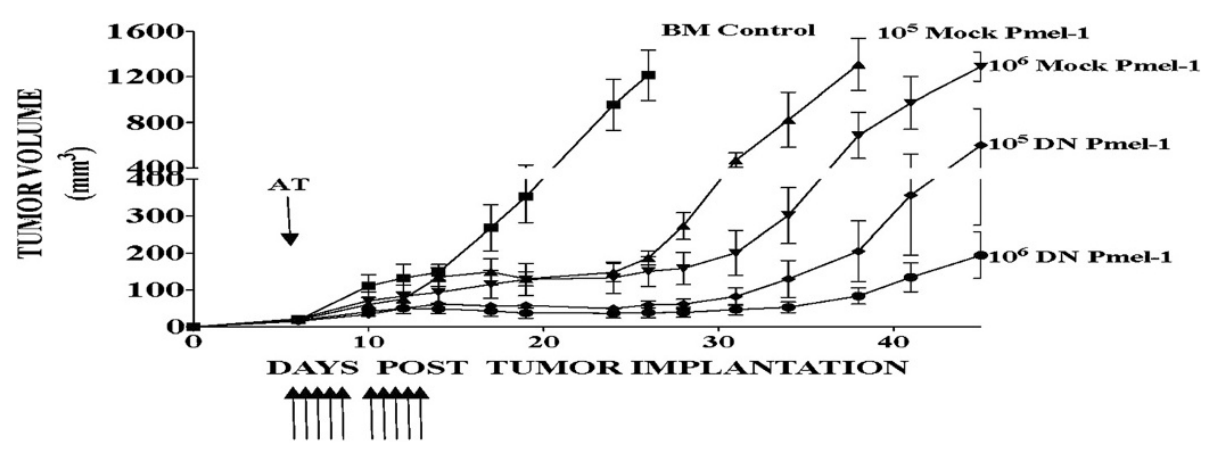

Figure 2 TGF $\beta$ insensitive Pmel-1 cells more effectively mediate B16 tumor regression. B16 tumor-bearing mice were conditioned with 900 CGy irradiation and given bone marrow support prior to adoptive transfer of either $10^{5}$ or $10^{6}$ mock or DN transduced Pmel-1 and IL2 support. Compared to mock transduced Pmel-1, DN Pmel-1 demonstrate better anti-tumor activity. Experiment was performed twice with similar results.

suggesting that these engineered $\mathrm{T}$ cells are more biologically active in the tumor microenvironment.

TGF $\beta$ is a pleiotropic cytokine which plays a significant role in various cellular processes including cellular proliferation, differentiation, and activation [8,25]. Multiple studies have demonstrated a direct correlation between TGF $\beta$ expression and tumor growth in melanoma and various other types of cancer [26]. TGF $\beta$ has an anti-proliferative effect and is considered to be a tumor suppressor during early stages of tumor development; however, during later stages of carcinogenesis, large amounts of TGF $\beta$ are secreted from many tumor types and have been implicated in immune evasion, mainly through negative regulation of immune effector function [27].

The DN TGF $\beta$ RII transgene has been demonstrated to be an effective strategy to circumvent the inhibitory effect of TGF $\beta$. Upon TGF $\beta$ binding to the extracellular region of the TGF $\beta$ receptor complex, intracellular kinase domain phosphorylates SMAD2 and SMAD3 protein, which translocate to the nucleus along with SMAD4, forming a complex that regulates gene expression. DN TGF $\beta$ receptor II lacks the intracellular kinase domain thus inhibiting intracellular signaling upon TGF $\beta$ binding. Mice with T cells that express this DN receptor as a transgene could reject various murine tumors including lymphoma and melanoma [16]. These elegant experiments have shown enhanced cross-presentation of tumor antigen and expansion of antigen-reactive $\mathrm{T}$ cells. Apart from inhibiting $\mathrm{T}$ cell effector function, TGF $\beta$ has an anti-proliferative effect. In vitro, exogenous TGF $\beta$ inhibits proliferation of wildtype $\mathrm{T}$ cells while $\mathrm{DN}$ transduced $\mathrm{T}$ cells retain proliferative and lytic function [12-14]. These TGF $\beta$ DN transgenic mice, at 12 wks, develop lethal multi-organ autoimmune disease $[8,9]$. This finding underscores the key role of TGF $\beta$ in Treg maintenance of peripheral tolerance.

Several reports have demonstrated the ability of adoptively transferred DN CD8+ T cells to mount potent anti- tumor response against solid tumors. The anti-tumor response is mainly attributed to increased immune effector function of adoptively transferred $\mathrm{T}$ cells. Whereas wildtype or TGF $\beta$ sensitive $\mathrm{T}$ cells are rendered anergic by tumor secreting TGF $\beta$, T cells that are insensitive to TGF $\beta$ signaling retain their cytolytic function and demonstrate the ability to produce IFN- $\gamma$ as well as granule exocytosis [12-15].

Natural killer and dendritic cells are also negatively regulated by TGF $\beta$. TGF $\beta$ inhibits expression of MHC II, costimulatory molecules, and cytokine production by dendritic cells. Tian et al. demonstrated that blockade of TGF $\beta$ signaling in dendritic cells leads to enhanced antitumor activity in a murine renal carcinoma vaccine model [28].

Tumor-secreted TGF $\beta$ not only affects $T$ cells through direct inactivation and growth inhibition, but also plays a significant role in the maintenance and function of regulatory $T$ cells [29-31]. It is well established that TGF $\beta$ promotes generation of induced regulatory T cells upon TCR stimulation and supports their survival in the periphery. Chen et al. showed that regulatory $\mathrm{T}$ cell dependent inhibition of tumor-specific $\mathrm{CD} 8+\mathrm{T}$ cell mediated cytotoxicity requires the expression of TGF $\beta$ receptor, as CD8 $+\mathrm{T}$ cells incapable of TGF $\beta$ signaling were resistant to suppression by regulatory T cells [10].

TGFßDNRII-transduced $\mathrm{T}$ cells are currently being used in clinical trials for Epstein-Barr virus (EBV)associated malignancies Hodgkin and non-Hodgkin lymphoma [13,14]. In preclinical models, DNtransduced EBV-specific T cells have a functional advantage over unmodified T cells: they were resistant to TGF $\beta$-mediated inhibition of proliferation cytolytic activity and mediated superior antitumor activity in a murine tumor model.

In summary, we demonstrate that antigen-specific $\mathrm{T}$ cells rendered insensitive to TGF $\beta$ through retroviral transduction of a DN TGF $\beta$ receptor II gene mount a 

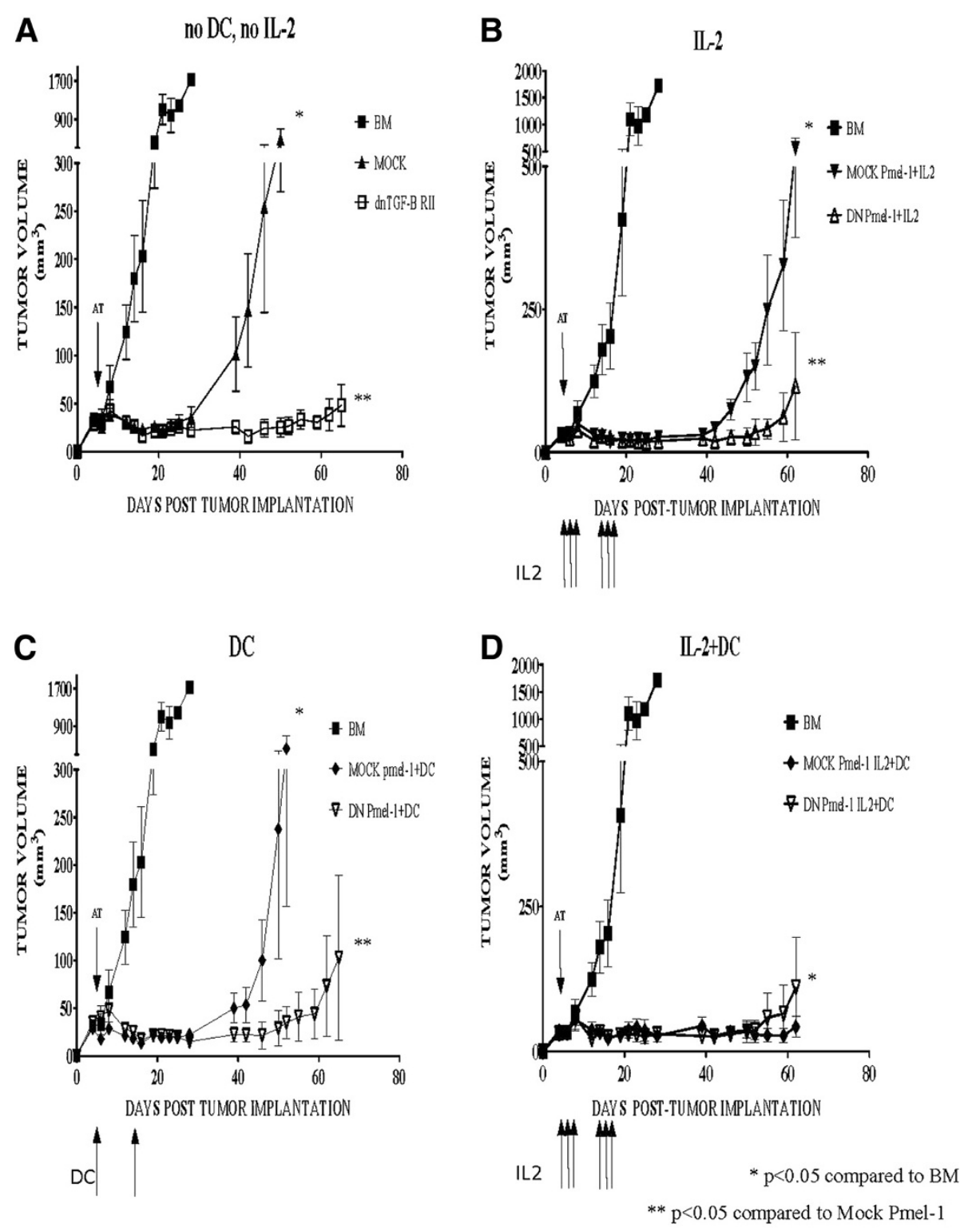

E

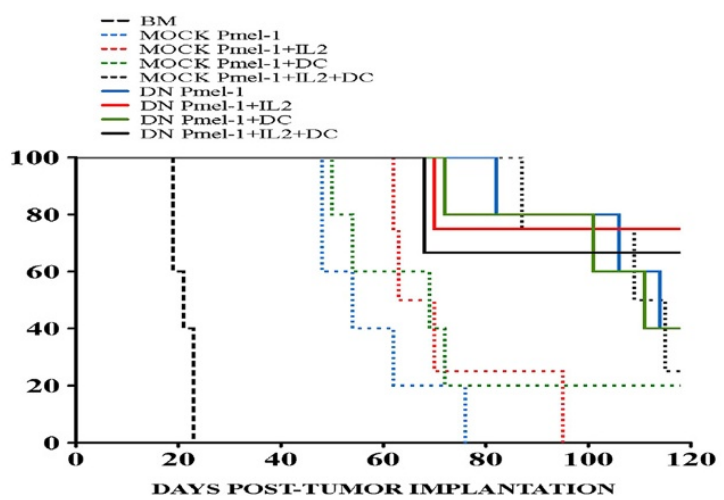


(See figure on previous page.)

Figure 3 DN Pmel-1 mediate tumor regression without IL2 and gp $100_{25-33} / \mathrm{DC}$ support. Mice bearing subcutaneous B16 tumors were myeloablated and given BM support prior to receiving adoptive transfer of $10^{6}$ mock or DN Pmel-1. Subsequently, mice received IL2 or gp100 25-33/DC support, both, or neither. Even without IL2 and gp100 $25-33$ /DC support, DN Pmel-1 mounted robust anti-tumor activity, repressing B16 tumor outgrowth up to 90 days post tumor implantation (3A). Support with IL2, gp100 $25-33$ /DC, or both lead to better tumor protection, however, DN Pmel-1 still demonstrate superior anti-tumor activity compared to mock Pmel-1 (3B, 3 C , 3D). E: Kaplan-Meier survival plot of mice treated as described above.

more effective anti-tumor response to B16 melanoma, most likely due to the ability of TGF $\beta$ insensitive T cells to remain active in the tumor microenvironment. $\mathrm{Nu}-$ merous studies have confirmed the presence of tumor infiltrating lymphocytes in resected cancer; however, these CD8+ $\mathrm{T}$ cells are functionally anergic when analyzed ex vivo [32]. Introducing a DN TGF $\beta$ RII gene into adoptively transferred $\mathrm{T}$ cells may prove to be an effective strategy against tumor-mediated inactivation of infiltrating lymphocytes.

\section{Endnote}

This work was supported by R01 CA129816, P01 CA132681, the Keck Foundation, and the Joy and Jerry Monkarsh Research Fund.
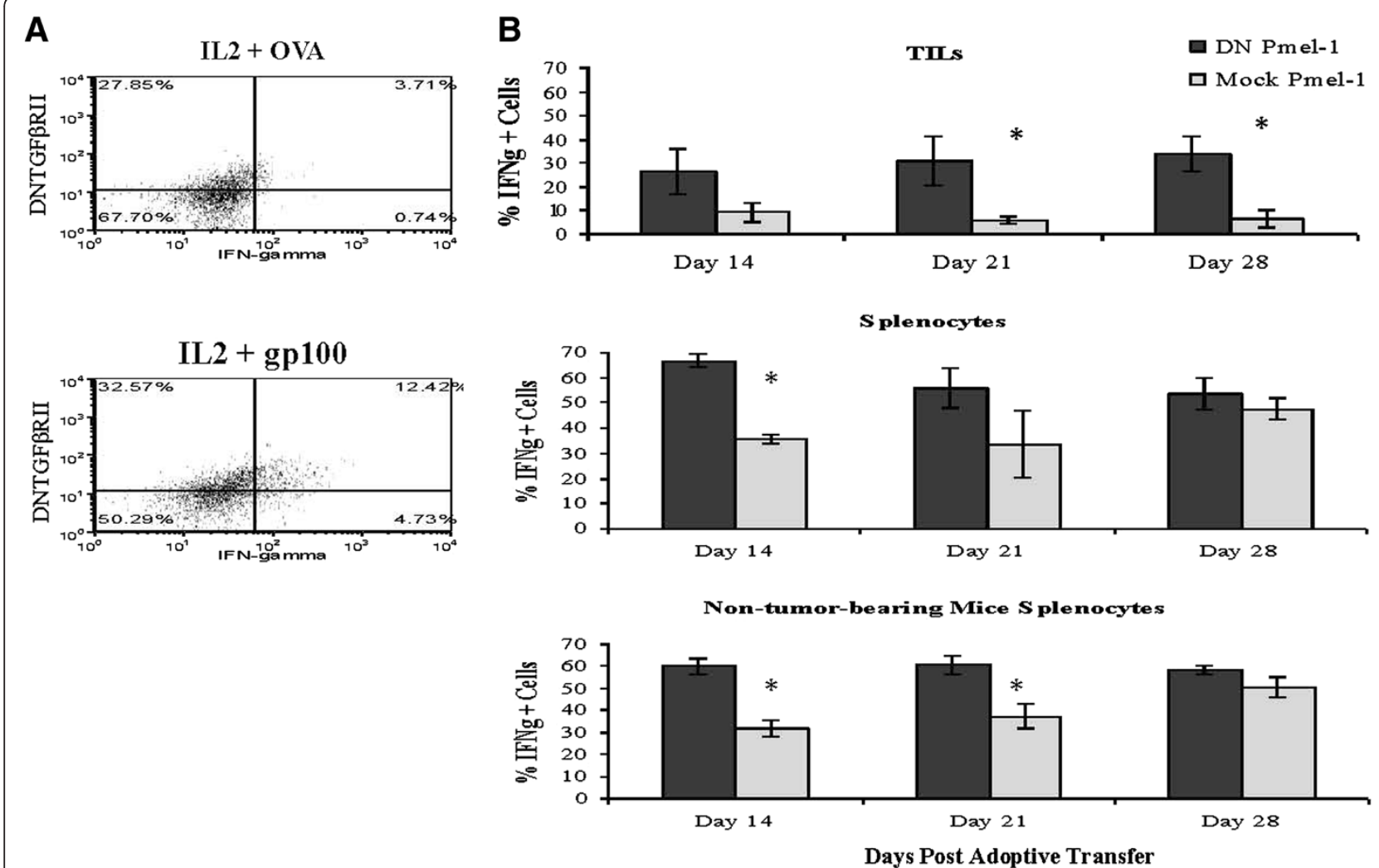

Figure 4 TGF $\beta$ insensitive Pmel-1 remain activated in immunosuppressive tumor microenvironment. On day 14,21 , and 28 post coadoptive transfer of DN and mock transduced Pmel-1 to B16 tumor-bearing and non tumor-bearing mice, splenocytes and tumor-infiltrating lymphocytes (TILs) of treated mice were analyzed for IFN-y production by intracellular cytokine staining. Between $2 \times 10^{4}-3 \times 10^{5} \mathrm{TILS}_{\text {and }} 1 \times 10^{7}-$ $6 \times 10^{7}$ splenocytes were collected on each day of experimentation. Figure $4 \mathbf{A}$ shows representative dot plots of TILs harvested from DN/mock mixture pmel-1 injected mice stimulated with either IL2 + control OVA peptide or IL2 + melanoma specific gp $100_{25-33}$ peptide. About 25-35\% of DN TGF $\beta$ RII tumor-infiltrating lymphocytes were actively secreting IFN- $\gamma$ upon re-stimulation compared to about $10 \%$ of mock-transduced TILs (4B). In both tumor-bearing and non tumor-bearing mice (all mice received DC vaccinations), a higher \% of DN Pmel-1 splenocytes produce IFNY on day 14 and 21 post adoptive transfer compared to mock Pmel-1 splenocytes (4B). Experiment was performed twice with similar results. 


\section{Additional file}

Additional file 1: Figure S1. Absolute cell counts of MOCK Pmel-1 and DN Pmel-1 cells in the spleen and in the tumor in the conditions described in Figure 4.

\section{Abbreviations}

TGFB: Transforming growth factor beta; IL2: Interleukin 2; TILs: Tumorinfiltrating lymphocytes; DC: Dendritic cells; TCR: T cell receptor.

\section{Competing interest}

The authors declare that they have no competing interests.

\section{Authors' contributions}

$J Q, Y W, D V, L F, A J$ and RK conducted animal experiments and analyses, contributed to experimental design and writing. WM, TC and JE contributed to experimental design and writing. All authors read and approved the final manuscript.

\section{Author details}

'Departments of Surgery, University of California, Los Angeles, CA 90095, USA. ${ }^{2}$ Radiation Oncology, University of California, Los Angeles, CA 90095, USA. ${ }^{3}$ Pediatrics, § Microbiology, Immunology and Molecular Genetics, University of California, Los Angeles, CA 90095, USA. ${ }^{4}$ Molecular and Medical Pharmacology, University of California, Los Angeles, CA 90095, USA. ${ }^{5}$ Jonsson Comprehensive Cancer Center, David Geffen School of Medicine, University of California, Los Angeles, CA 90095, USA. 'Division of Surgical Oncology, UCLA David Geffen School of Medicine, 54-140 CHS, Box 957182, 10833 LeConte Ave Los Angeles, CA 90095-1782, USA.

Received: 18 October 2011 Accepted: 13 April 2012

Published: 19 June 2012

\section{References}

1. Dudley ME, et al: CD8+ enriched "young" tumor infiltrating lymphocytes can mediate regression of metastatic melanoma. Clin Cancer Res 2010, 16(24):6122-6131.

2. Goff SL, et al: Tumor infiltrating lymphocyte therapy for metastatic melanoma: analysis of tumors resected for TIL. J Immunother 2010, 33(8):840-847.

3. Rosenberg SA, et al: Adoptive cell transfer: a clinical path to effective cancer immunotherapy. Nat Rev Cancer 2008, 8(4):299-308.

4. Gajewski TF: Failure at the effector phase: immune barriers at the level of the melanoma tumor microenvironment. Clin Cancer Res 2007, 13(18 Pt 1):5256-5261.

5. Wrzesinski SH, Wan YY, Flavell RA: Transforming growth factor-beta and the immune response: implications for anticancer therapy. Clin Cancer Res 2007, 13(18 Pt 1):5262-5270.

6. Leivonen SK, Kahari VM: Transforming growth factor-beta signaling in cancer invasion and metastasis. Int J Cancer 2007, 121(10):2119-2124.

7. Rubtsov YP, Rudensky AY: TGFbeta signalling in control of T-cell-mediated self-reactivity. Nat Rev Immunol 2007, 7(6):443-453.

8. Fahlen $L$, et al: $T$ cells that cannot respond to TGF-beta escape control by CD4(+)CD25(+) regulatory T cells. J Exp Med 2005, 201(5):737-746.

9. Carrier $Y$, et al: Th3 cells in peripheral tolerance. II. TGF-beta-transgenic Th3 cells rescue IL-2-deficient mice from autoimmunity. J Immunol 2007, 178(1):172-178.

10. Chen ML, et al: Regulatory $\mathrm{T}$ cells suppress tumor-specific CD8 T cell cytotoxicity through TGF-beta signals in vivo. Proc Natl Acad Sci U S A 2005, 102(2):419-424.

11. Li MO, Sanjabi S, Flavell RA: Transforming growth factor-beta controls development, homeostasis, and tolerance of T cells by regulatory T celldependent and -independent mechanisms. Immunity 2006, 25(3):455-471.

12. Bollard $\mathrm{CM}$, et al: Adapting a transforming growth factor beta-related tumor protection strategy to enhance antitumor immunity. Blood 2002, 99(9):3179-3187.

13. Foster $A E$, et al: Antitumor activity of EBV-specific T lymphocytes transduced with a dominant negative TGF-beta receptor. J Immunother 2008, 31(5):500-505.
14. Lacuesta $K$, et al: Assessing the safety of cytotoxic T lymphocytes transduced with a dominant negative transforming growth factor-beta receptor. J Immunother 2006, 29(3):250-260.

15. Wang $L$, et al: Immunotherapy for human renal cell carcinoma by adoptive transfer of autologous transforming growth factor betainsensitive CD8+ T cells. Clin Cancer Res 2010, 16(1):164-173.

16. Gorelik L, Flavell RA: Immune-mediated eradication of tumors through the blockade of transforming growth factor-beta signaling in T cells. Nat Med 2001, 7(10):1118-1122.

17. Overwijk WW, et al: Tumor regression and autoimmunity after reversal of a functionally tolerant state of self-reactive CD8+ T cells. J Exp Med 2003, 198(4):569-580.

18. Wieser R, et al: Signaling activity of transforming growth factor beta type II receptors lacking specific domains in the cytoplasmic region. $\mathrm{Mol}$ Cell Biol 1993, 13(12):7239-7247.

19. Koya RC, et al: Kinetic phases of distribution and tumor targeting by $T$ cell receptor engineered lymphocytes inducing robust antitumor responses. Proc Natl Acad Sci U S A 2010, 107(32):14286-14291.

20. Ribas A, et al: Genetic immunization for the melanoma antigen MART-1/ Melan-A using recombinant adenovirus-transduced murine dendritic cells. Cancer Res 1997, 57(14):2865-2869.

21. Ribas $A$, et al: Enhanced tumor responses to dendritic cells in the absence of CD8-positive cells. J Immunol 2004, 172(8):4762-4769.

22. Craft N, et al: The TLR7 agonist imiquimod enhances the anti-melanoma effects of a recombinant Listeria monocytogenes vaccine. J Immunol 2005, 175(3):1983-1990.

23. Vo DD, et al: Enhanced antitumor activity induced by adoptive T-cell transfer and adjunctive use of the histone deacetylase inhibitor LAQ824. Cancer Res 2009, 69(22):8693-8699.

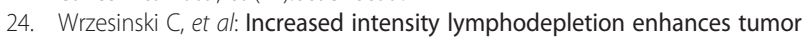
treatment efficacy of adoptively transferred tumor-specific $T$ cells. $\mathrm{J}$ Immunother 2010, 33(1):1-7.

25. Kano MR, et al: Improvement of cancer-targeting therapy, using nanocarriers for intractable solid tumors by inhibition of TGF-beta signaling. Proc Natl Acad Sci U S A 2007, 104(9):3460-3465.

26. Penafuerte C, Galipeau J: TGF beta secreted by B16 melanoma antagonizes cancer gene immunotherapy bystander effect. Cancer Immunol Immunother 2008, 57(8):1197-1206.

27. Javelaud D, Alexaki VI, Mauviel A: Transforming growth factor-beta in cutaneous melanoma. Pigment Cell Melanoma Res 2008, 21(2):123-132.

28. Tian F, et al: Vaccination with transforming growth factor-beta insensitive dendritic cells suppresses pulmonary metastases of renal carcinoma in mice. Cancer Lett 2008, 271(2):333-341.

29. Gorelik L, Constant S, Flavell RA: Mechanism of transforming growth factor beta-induced inhibition of T helper type 1 differentiation. J Exp Med 2002, 195(11):1499-1505.

30. Li MO, Wan YY, Flavell RA: T cell-produced transforming growth factorbeta1 controls $T$ cell tolerance and regulates Th1- and Th17-cell differentiation. Immunity 2007, 26(5):579-591.

31. Horwitz DA, et al: Critical role of IL-2 and TGF-beta in generation, function and stabilization of Foxp3 + CD4+ Treg. Eur J Immunol 2008, 38(4):912-915.

32. Harlin $\mathrm{H}$, et al: Tumor progression despite massive influx of activated CD8(+) T cells in a patient with malignant melanoma ascites. Cancer Immunol Immunother 2006, 55(10):1185-1197.

\section{doi:10.1186/1479-5876-10-127}

Cite this article as: Quatromoni et al.: T cell receptor (TCR)-transgenic CD8 lymphocytes rendered insensitive to transforming growth factor beta (TGF $\beta$ ) signaling mediate superior tumor regression in an animal model of adoptive cell therapy. Journal of Translational Medicine 2012 10:127. 